\title{
Chlamydial SET domain protein functions as a histone methyltransferase
}

\author{
Masayuki Murata, ${ }^{1}$ Yoshinao Azuma, ${ }^{1}$ Koshiro Miura, ${ }^{1,2} \dagger$ \\ Mohd. Akhlakur Rahman, ${ }^{1}$ Minenosuke Matsutani, ${ }_{1}^{1}$ Masahiro Aoyama, ${ }^{1}$ \\ Harumi Suzuki, ${ }^{1}$ Kazuro Sugi ${ }^{2}$ and Mutsunori Shirai ${ }^{1}$ \\ ${ }^{1}$ Department of Microbiology and Immunology, Yamaguchi University School of Medicine, 1-1-1 \\ Minami-Kogushi, Ube, Yamaguchi 755-8505, Japan \\ ${ }^{2}$ Department of Clinical Research, National Sanyo Hospital, 685 Higashi-Kiwa, Ube, Yamaguchi \\ 755-0241, Japan
}

Correspondence

Yoshinao Azuma

yazuma@yamaguchi-u.ac.jp

Mutsunori Shirai

mshirai@yamaguchi-u.ac.jp

Received 12 June 2006

Revised 20 October 2006

Accepted 20 October 2006

\begin{abstract}
SET domain genes have been identified in numbers of bacterial genomes based on similarity to SET domains of eukaryotic histone methyltransferases. Herein, a Chlamydophila pneumoniae SET domain gene was clarified to be coincidently expressed with $h c t A$ and $h c t B$ genes encoding chlamydial histone $\mathrm{H} 1$-like proteins, $\mathrm{Hc} 1$ and $\mathrm{Hc} 2$, respectively. The SET domain protein (cpnSET) is localized in chlamydial cells and interacts with $\mathrm{Hc} 1$ and $\mathrm{Hc} 2$ through the C-terminal SET domain. As expected from conservation of catalytic sites in cpnSET, it functions as a protein methyltransferase to murine histone $\mathrm{H} 3$ and $\mathrm{Hc} 1$. However, little is known about protein methylation in the molecular pathogenesis of chlamydial infection. cPnSET may play an important role in chlamydial cell maturation due to modification of chlamydial histone $\mathrm{H} 1$-like proteins.
\end{abstract}

\section{INTRODUCTION}

Chlamydophila pneumoniae is an obligatory intracellular eubacterium that causes acute respiratory diseases and may be involved in chronic inflammatory processes, such as atherosclerosis (Rosenfeld et al., 2000), asthma (Hahn et al., 1991) and Alzheimer's disease (Itzhaki et al., 2004). Persistence of chlamydial infection has been thought to be important for chronic diseases and has been characterized using model animals and activation stimuli such as cytokines and antibiotics (Beatty et al., 1993; Belland et al., 2003; Malinverni et al., 1995; Mehta et al., 1998). However, molecular-level relationships between chronic disease progression and persistent infection are not yet clear.

Chlamydiae exhibit a unique life cycle in which they alternate morphologies between elementary bodies (EBs) and reticulate bodies (RBs). EBs are transcriptionally inactive electron-dense particles that are internalized into host cells by inducing phagocytosis. EB differentiation into RBs occurs with the development of phagosomes

tPresent address: Department of Veterinary Biosciences, the Ohio State University, 1900 Coffey Rd, Columbus, OH 43210, USA.

Abbreviations: cPnSET, Chlamydophila pneumoniae SET domain protein; EB, elementary body; RB, reticulate body; FCS, fetal calf serum; GST, glutathione S-transferase; h.p.i., hours post-infection.

A supplementary table and three supplementary figures are available with the online version of this paper. into inclusions. Transcriptionally active RBs multiply by binary fission with nutrients acquired from the host cell. At the end of the developmental cycle, RBs are converted into EBs and released from host cells for the next infection. Besides the developmental cycle, during persistent infection caused by exposure to interferon gamma (IFN- $\gamma$ ) or antibiotics, RBs differentiate into aberrantly large and non-multiplying RBs (Belland et al., 2003). However, little is known about the switching mechanism whereby vegetative RBs convert into infectious EBs or aberrant RBs. Understanding this molecular system should be helpful for the prevention of persistent chlamydial infection.

Two eukaryotic histone H1-like proteins of chlamydiae, Hc1 and $\mathrm{Hc} 2$, are present mainly in EBs, where those proteins bind DNA and promote genomic DNA condensation (Barry et al., 1992; Hackstadt et al., 1991; Perara et al., 1992; Tao et al., 1991). Recently a small regulatory RNA gene was identified as a suppressor of the lethal phenotype of hctA overexpression in Escherichia coli and it was shown to negatively regulate $\mathrm{Hcl}$ synthesis at an early stage of infection (Grieshaber et al., 2006). These histone-like proteins may act as global transcriptional regulators and play a critical role for the transformation of vegetative RBs into infectious EBs. Transcriptional, translational and functional regulations of $\mathrm{Hcl}$ and $\mathrm{Hc} 2$ may be important for the morphological switching. Chlamydial genome analyses have revealed the existence of another candidate gene as a regulator of $\mathrm{Hc} 1$ and $\mathrm{Hc} 2$, termed the set gene, 
which encodes a protein containing a domain similar to the eukaryotic SET domain (Stephens et al., 1998). Eukaryotic SET domains were initially identified in the C-terminal ends of Drosophila transcriptional regulatory factors (AlvarezVenegas \& Avramova, 2002; Jones \& Gelbart, 1993; Kouzarides, 2002; Kuzmichev et al., 2005) and have been shown to be involved in chromatin remodelling due to histone methyltransferase activity to specific residues in amino-terminal histone tails, such as histone $\mathrm{H} 3 \mathrm{~K} 9$ and K27 (Marmorstein, 2003.; Xiao et al., 2003).

Herein, we demonstrate that the chlamydial SET domain protein physically interacts with chlamydial histone-like proteins $\mathrm{Hcl}$ and $\mathrm{Hc} 2$, and functions as a histone methyltransferase to methylate mouse histone $\mathrm{H} 3$ and Hcl. The results suggest involvement of the SET domain protein in chlamydial cell transformation from RBs to EBs.

\section{METHODS}

Chemicals, cell line and bacterial strains. Gentamicin, cycloheximide, Hoechst 33258 and Dulbecco's modified Eagle medium were purchased from Sigma-Aldrich. Fetal calf serum (FCS) was from Cansera International. Anti-C. pneumoniae-specific monoclonal antibody (RR402) was purchased from Washington Research Foundation. FITC-conjugated goat anti-mouse antibody and Alexa 545-conjugated goat anti-rabbit antibody were obtained from Invitrogen. S-Adenosyl-[methyl- $\left.{ }^{14} \mathrm{C}\right]$-L-methionine was purchased from Amersham.

HEp-2 cells (ATCC CCL-23) were used as host cells for infection by $C$. pneumoniae J138, isolated in Japan in 1994 (Shirai et al., 2000). C. pneumoniae $\mathrm{J} 138 \mathrm{EBs}$ were purified by sucrose-gradient centrifugation and stored at $-80^{\circ} \mathrm{C}$ in SPG buffer ( $\mathrm{pH} 7.2$ ), which consists of $250 \mathrm{mM}$ sucrose, $10 \mathrm{mM}$ sodium phosphate and $5 \mathrm{mM}$ glutamate. Chlamydial titres were adjusted to $2.0 \times 10^{8}$ inclusion-formation units (i.f.u.) $\mathrm{ml}^{-1}$.

Chlamydial infection. Chlamydial infections were performed by methods described previously (Rahman et al., 2005). Briefly, HEp-2 cells were grown in HEp-2 medium (Dulbecco's modified Eagle medium supplemented with $10 \%$ heat-inactivated FCS and $50 \mu \mathrm{g}$ gentamicin $\mathrm{ml}^{-1}$ ) at $37^{\circ} \mathrm{C}, 5 \% \mathrm{CO}_{2}$. Prior to infection, $2.0 \times 10^{5}$ HEp-2 cells were seeded to each well of six-well tissue culture plates and allowed to adhere for $24 \mathrm{~h}$. Infection was performed by addition of C. pneumoniae $\mathrm{J} 138 \mathrm{EBs}$ at 0.20 m.o.i., followed by centrifugation at $22^{\circ} \mathrm{C}$ for $60 \mathrm{~min}$ at $700 \mathrm{~g}$. After incubation for $30 \mathrm{~min}$ at $36^{\circ} \mathrm{C}$, $5 \% \mathrm{CO}_{2}$, the inocula were replaced with post-infection medium (Dulbecco's modified Eagle medium with $5 \%$ heat-inactivated FCS, $50 \mu \mathrm{g}$ gentamicin $\mathrm{ml}^{-1}$ and $1 \mu \mathrm{g}$ cycloheximide $\mathrm{ml}^{-1}$ ). The infectants were incubated for up to $72 \mathrm{~h}$ at $36^{\circ} \mathrm{C}, 5 \% \mathrm{CO}_{2}$.

Vector construction. pGEX $(2 \mathrm{~T}-\mathrm{P})+\mathrm{cpnSET}$ full-length and pGEX (2T-P) + cpnSET (206-219 aa) were constructed by cloning the C. pneumoniae J138 set gene fragments into pGEX(2T-P) (Azuma et al., 1995). Three deletion series of pGEX(2T-P) + Hc1 [Hc1-1 (aa 1-50), Hcl-2 (aa 41-78) and Hc1-3 (aa 65-123)] were constructed based on the pGEX $(2 \mathrm{~T}-\mathrm{P})+\mathrm{Hcl}$. For the yeast twohybrid study, pGBKT7 + cpnSET was prepared by cloning C. pneumoniae J138 set gene into pGBKT7 (Clontech). For construction of pGADT7 + Hc1 and pGADT7 + Hc2, C. pneumoniae J138 hctA and $h c t B$ genes, respectively, were cloned into pGADT7 (Clontech). Eight deletion series of pGBKT7-cpnSET were constructed by PCR using pGBKT7-cpnSET. pGEX4T-3-mG9a and pGEX4T-3-H3, encoding GST fusion G9a (621-1000 aa) and GST fusion histone H3 (1-50 aa), respectively, were kind gifts of Professor Yoichi Shinkai
(Kyoto University, Kyoto, Japan) (Tachibana et al., 2001). All primers used in this work are shown in Supplementary Table S1, available with the online version of this paper.

Preparation of recombinant proteins and anti-cpnSET antiserum. GST fusion proteins were produced in E. coli JM109 cells and purified using glutathione-agarose affinity purification in lysis buffer [20 mM Tris/HCl ( $\mathrm{pH} 8.0), 5 \mathrm{mM}$ EDTA, $0.5 \%$ Triton X100, $0.2 \mathrm{mM}$ PMSF and a protease inhibitor mixture] (Azuma et al., 1993). One milligram of GST fusion cpnSET protein was cleaved with $0.02 \mathrm{U}$ thrombin (Novagen) in thrombin reaction solution [20 mM Tris/HCl (pH 8.4), $150 \mathrm{mM} \mathrm{NaCl}, 2.5 \mathrm{mM} \mathrm{CaCl}_{2}$ ] for $2 \mathrm{~h}$ at $20^{\circ} \mathrm{C}$, and the thrombin was removed by incubation with $50 \mu \mathrm{l}$ $p$-aminobenzamidene-agarose beads (Amersham) for $1 \mathrm{~h}$ at $4{ }^{\circ} \mathrm{C}$. Anti-cpnSET rabbit polyclonal sera were prepared by immunization of rabbits five times every other week with $0.1 \mu \mathrm{g}$ of the purified GST fusion cpnSET (aa 206-219) protein, following the method described previously (Miura et al., 2001).

Histochemical analysis. After fixation with $100 \%$ methanol for $60 \mathrm{~min}$, the infectants were incubated with anti-C. pneumoniae-specific monoclonal antibody (RR402) and anti-cpnSET rabbit serum as described above for $60 \mathrm{~min}$ at $25^{\circ} \mathrm{C}$. After washing, cells were stained with FITC-conjugated goat anti-mouse antibody and Alexa 545-conjugated goat anti-rabbit antibody. Nucleic acids were stained with $2 \mu \mathrm{g}$ Hoechst $33258 \mathrm{ml}^{-1}$ for $10 \mathrm{~min}$. Microscopic observation was performed with an LSM510 laser scanning confocal microscope (Zeiss).

Quantitative RT-PCR. For quantitative RT-PCR by a LightCycler (Roche), QuantiTect SYBR Green RT-PCR (Qiagen) was used with a total RNA fraction extracted from C. pneumoniae-infected cells. Reactions were performed based on the manufacturer's instructions. All primers are shown as a supplementary table within the online version of this paper at Table $\mathrm{S} 1$ for primers.

Protein interaction analyses. Yeast two-hybrid analysis was performed using MatchMaker GAL4 Two-Hybrid System 3 kits (Clontech) according to the manufacturer's instructions. Transformants were assayed by growth on plates without leucine, tryptophan and histidine, and without leucine and tryptophan as a control.

Structure modelling of cpnSET was carried out using the virus SET structure as a template (Eswar et al., 2003; Manzur et al., 2003) and peptide docking analysis onto cpnSET was performed using AutoDock (Morris et al., 1996).

In vitro histone methyltransferase assay. Procedures for in vitro measurement of histone methyltransferase activity were adapted from the protocol reported previously (Tachibana et al., 2001). Briefly, the assay was carried out with $0.5 \mu \mathrm{g} \mathrm{mG9a}$ or cpnSET protein and $0.5 \mu \mathrm{g}$ GST fusion mouse histone $\mathrm{H} 3$ or chlamydial $\mathrm{Hcl}$ as a substrate in $50 \mu \mathrm{l}$ reaction buffer $(50 \mathrm{mM}$ Tris/HCl $\mathrm{pH} 8.5,20 \mathrm{mM} \mathrm{KCl}, 10 \mathrm{mM} \mathrm{MgCl} 2,10 \mathrm{mM} \beta$-mercaptoethanol, $250 \mathrm{mM}$ sucrose and $4.6 \mathrm{kBq}$ S-adenosyl-[methyl- $\left.{ }^{14} \mathrm{C}\right]-\mathrm{L}$-methionine as methyl donor). After incubation for $60 \mathrm{~min}$ at $37^{\circ} \mathrm{C}$, reactions were stopped by addition of $15 \mu \mathrm{l}$ SDS buffer [6\% SDS, $150 \mathrm{mM}$ Tris/HCl (pH 6.8), $300 \mathrm{mM}$ DTT, $0.1 \%$ BPB and $30 \%(\mathrm{v} / \mathrm{v})$ glycerol] and boiling at $100{ }^{\circ} \mathrm{C}$ for $10 \mathrm{~min}$. Methyl- ${ }^{14} \mathrm{C}$ was detected using a BAS-2000 scanner (FujiFilm) after protein separation by $12 \%$ acrylamide SDS-PAGE.

\section{RESULTS}

\section{Chlamydial SET domain protein}

Chlamydial genome analysis has revealed the existence of a number of genes similar to eukaryotic genes involved in 
(a)

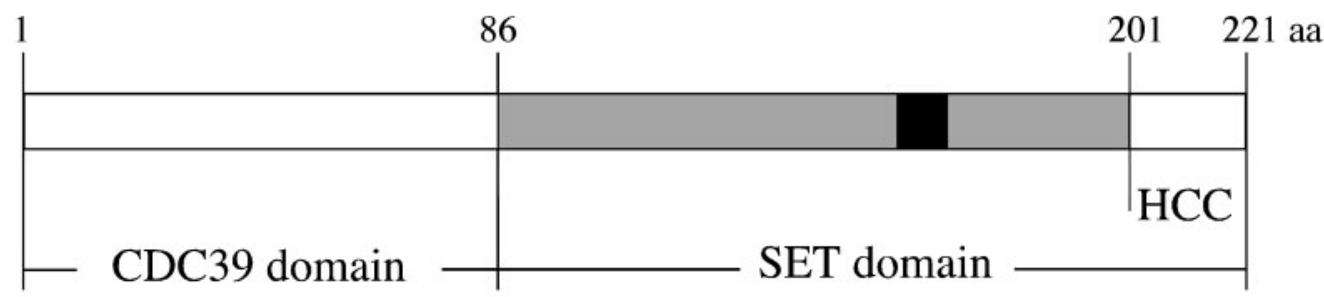

(b)

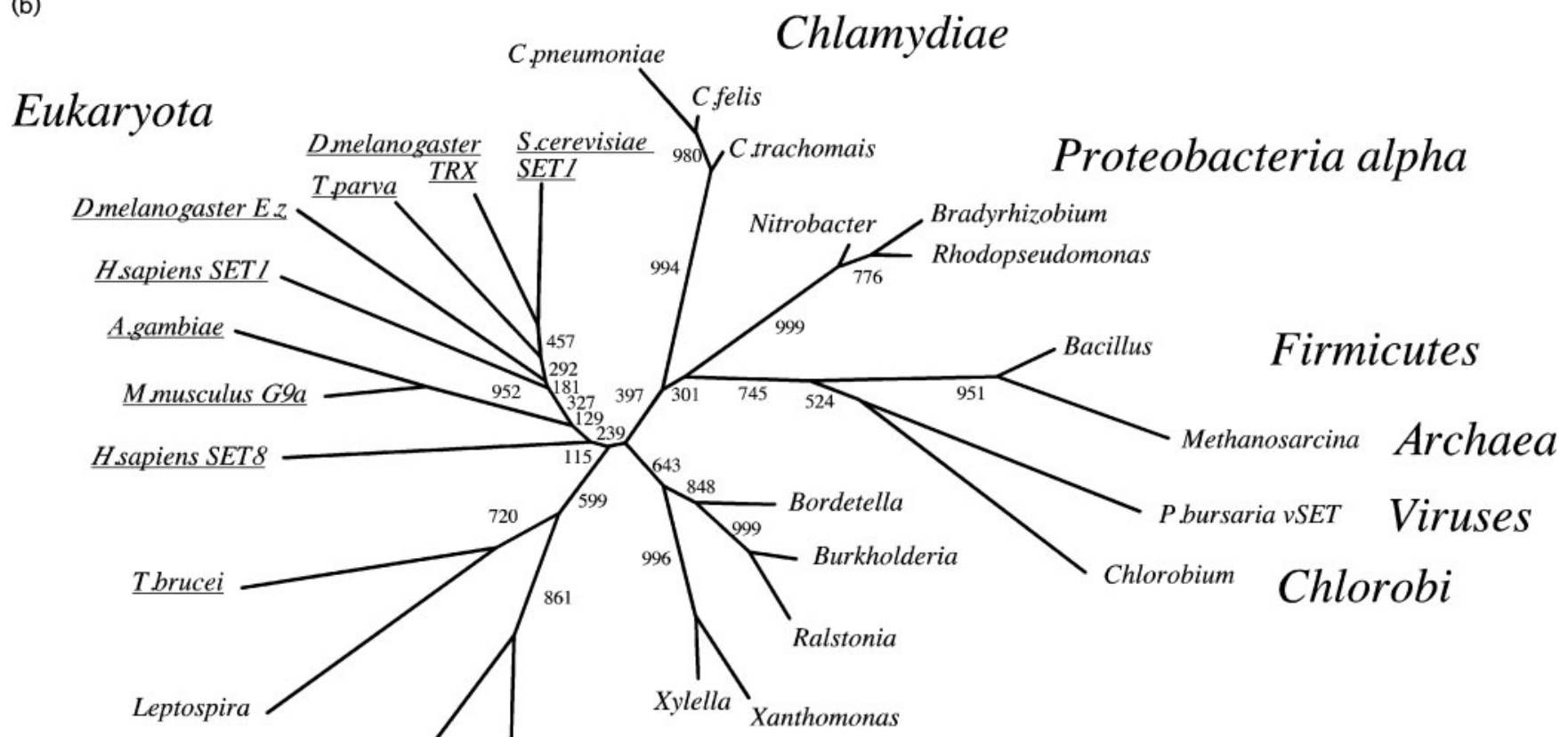

\section{Spirochaetes}

Silicibacter

\section{Proteobacteria beta and gamma}

\section{Proteobacteria alpha}

Fig. 1. C. pneumoniae SET domain protein (cpnSET). (a) Domains in cpnSET domain protein. The signature motif, 'RFINHXCXPN' (Dillon et al., 2005) is indicated with a black box. The HCC in the C-terminus shows a highly charged and conserved (HCC) domain. (b) Phylogenetic analysis of SET-domain proteins based on sequence alignment. The alignment includes the known members of the SET-domain protein family. C. pneumoniae, Chlamydophila pneumoniae J138 (NP300935); C. felis, Chlamydophila felis Fe/C-56 (CF0125); C. trachomatis, Chlamydia trachomatis D/UW-3/CX (NP220256); Bradyrhizobium, Bradyrhizobium japonicum USDA110 (BAC51052); Rhodopseudomonas, Rhodopseudomonas palustris BisA53 (ZP00807683); Nitrobacter, Nitrobacter winogradskyi Nb-255 (ABA05415); Bacillus, Bacillus anthracis Ames (ZP00390019); Burkholderia, Burkholderia cenocepacia (ZP00459508); Ralstonia, Ralstonia eutropha JMP134 (AAZ62749); Bordetella, Bordetella pertussis 12822 (CAE44800); Xanthomonas, Xanthomonas oryzae MAFF (YP200046); Xylella, Xylella fastidiosa (AAF84287); Mesorhizobium, Mesorhizobium loti MAFF303099 (BAB50081); Silicibacter, Silicibacter pomeroyi DSS-3 (AAV95586); Chlorobium, Chlorobium tepidum TLS (AAM72187); Leptospira, Leptospira interrogans serovar Copenhageni str. (AAS71524); Methanosarcina, Methanosarcina mazei Go1 (AAM32541); P. bursaria vSET, Paramecium bursaria chlorella virus-1 (NP048968); H. sapiens SET1, Homo sapiens (NP071900); H. sapiens SET8, Homo sapiens (NP065115); M. musculus G9a, Mus musculus (NP665829); D. melanogaster TRX, Drosophila melanogaster (NP599109); D. melanogaster E.z., Drosophila melanogaster (AAF50149); S. cerevisiae SET1, Saccharomyces cerevisiae S288C (P38827); T. parva, Theileria parva Muguga (AN32159); T. brucei, Trypanosoma brucei TREU927/4 (EAN77879); A. gambiae, Anopheles gambiae PEST (EAA07914). 
(a)
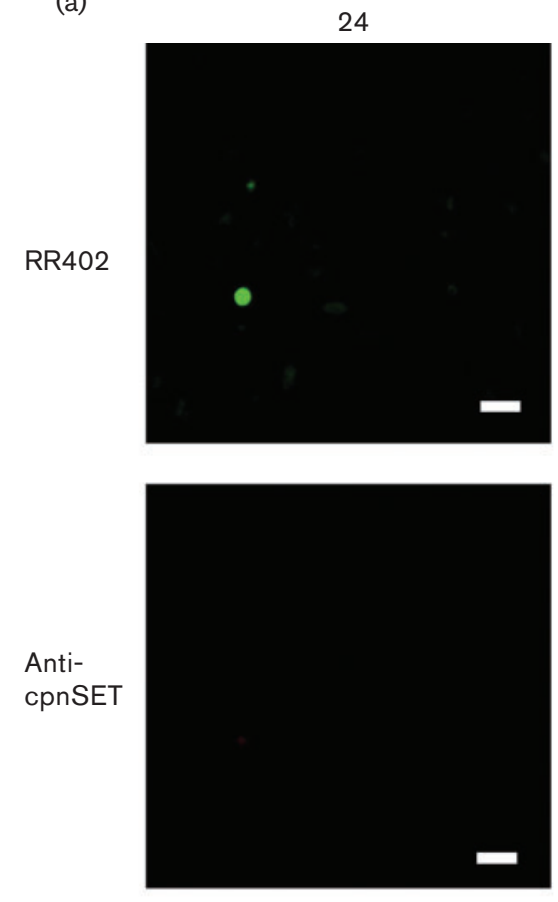

(b)

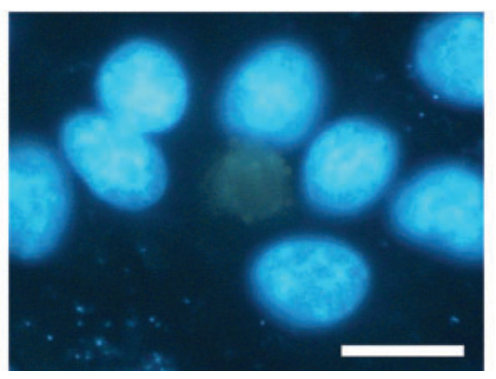

60
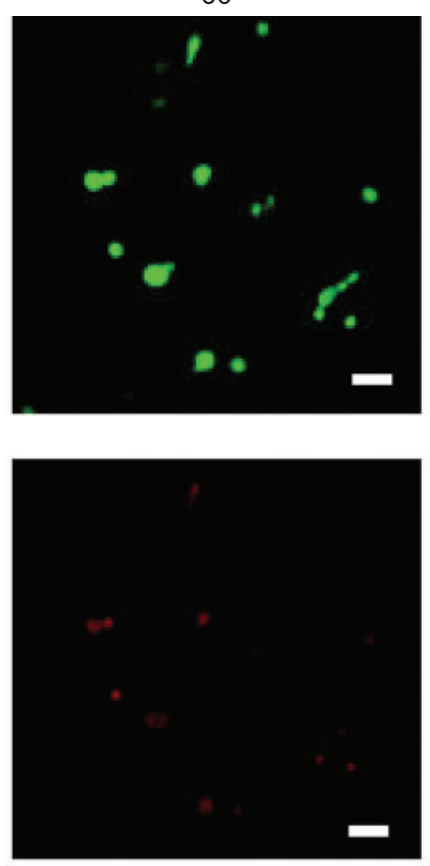

72
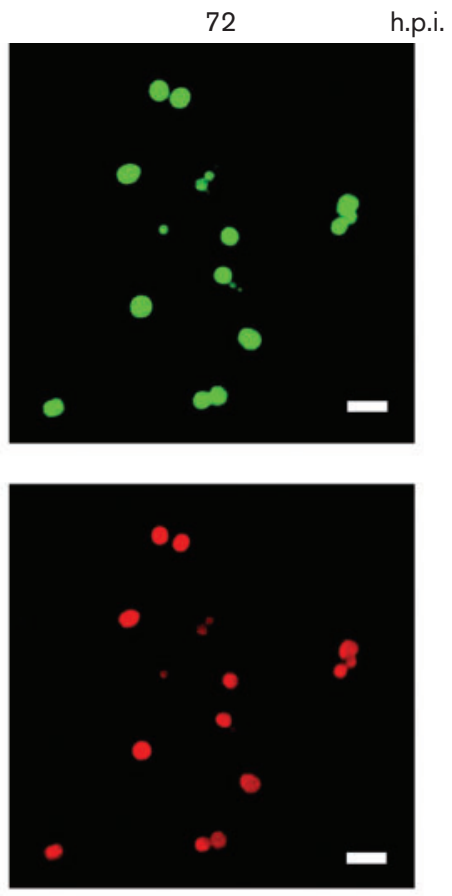

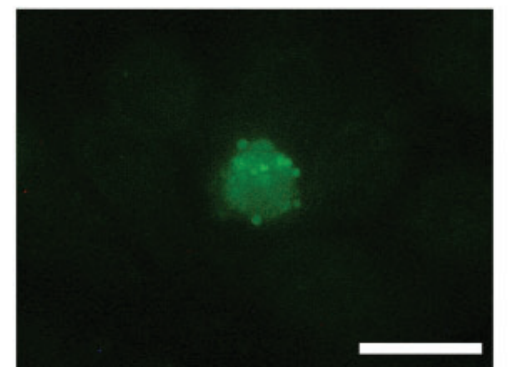

Fig. 2. Expression and localization of cpnSET within C. pneumoniae J138-infected HEp-2 cells. (a) Infected cells were fixed at 24, 60 and 72 h.p.i. and stained with the indicated antibodies. The anti-cpnSET is C. pneumoniae SET-specific rabbit polyclonal antibody prepared in this work and RR402 is C. pneumoniae-specific murine monoclonal antibody used as a control. Magnification, $\times 400$; bars, $10 \mu \mathrm{m}$. (b) The left and right panels are Hoechst 33258 and anti-cpnSET rabbit serum staining, respectively. Magnification, $\times 1000$; bars, $5 \mu \mathrm{m}$.

chromatin maintenance, such as histone, SET and SWI/SNF (Azuma et al., 2006; Carlson et al., 2005; Kalman et al., 1999; Read et al., 2000, 2003; Shirai et al., 2000; Stephens et al., 1998; Thomson et al., 2005). CPj0878 is assigned as a gene coding a SET domain protein in C. pneumoniae J138 and well conserved (overall more than $60 \%$ identities) in the family Chlamydiaceae. The C-terminal SET domain shows approximately $30 \%$ identities to many eukaryotic SET domains. The eukaryotic SET domains are well characterized as catalytic domains of histone methyltransferases involved in chromatin remodelling, especially transcriptional regulation and establishment of heterochromatin (Marmorstein, 2003.; Xiao et al., 2003). The chlamydial SET domain protein consists of two distinct domains, CDC39 and SET (Fig. 1a). The N-terminal one-third is partially similar to a CDC39/NotI component in a yeast general transcription-negative regulator CCR4-Not complex involved in controlling mRNA initiation (Liu et al., 1998). This CDC39 region is well conserved only in chlamydial SET domain proteins but not in other organism SET proteins. The C-terminal two-thirds region preserves SET signature motifs, such as $S$-adenosyl-L-methionine-binding sites and a catalytic site for the histone methyltransferase activity (Zhang et al., 2003).

The chlamydial SET domain gene (set) was thought to be established as a result of horizontal gene transfer from a eukaryotic organism to a chlamydial ancestor (Stephens et al., 1998). But recent progress in genome analysis has revealed the existence of numbers of genes encoding SETlike domains in non-eukaryotic organisms, e.g. Paramecium bursaria chlorella virus-1 (Manzur et al., 2003), archaeal 
(a)

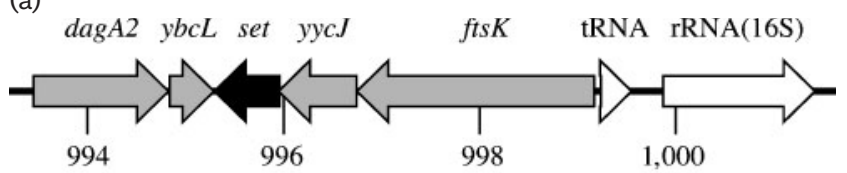

(b)

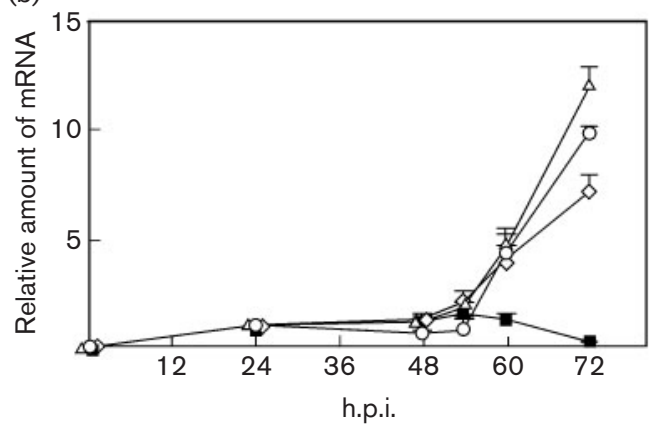

Fig. 3. C. pneumoniae set gene. (a) Genome map showing the set gene locus. (b) Quantitative RT-PCR of set, hctA, hctB and ompA genes. Total RNA was prepared from HEp-2 cells (ATCC CCL-23) infected with C. pneumoniae J138 (Rahman et al., 2005) at $0,12,24,48,54,60$ and 72 h.p.i. Infection was carried out as described previously (Rahman et al., 2005). Expression of set $(\bigcirc)$, hctA $(\triangle), \operatorname{hctB}(\diamond)$ and $\operatorname{ompA}(\square)$ genes were normalized with $16 \mathrm{~S}$ rRNA. Relative expression was calculated as the ratio to the value of the expression of each gene at $24 \mathrm{~h}$.

Methanosarcina mezei (Manzur \& Zhou, 2005) and a variety of eubacterial phyla such as Firmicutes, Proteobacteria, Chlorobi, Spirochaetes and Chlamydiae. Phylogenetic analysis of eubacterial and other SETs illustrates that eubacterial SETs diverge into a few groups similar to the general taxological phyla (Fig. 1b). It suggests that chlamydial set genes are not transferred horizontally from eukaryotic organisms, but rather likely diverged from a bacterial origin. The sequence alignment of SETs is shown as Supplementary Fig. S1 with the online version of this paper.

\section{Gene expression and protein localization of cpnSET}

Since the existence of the C. pneumoniae SET domain protein (cpnSET) was proposed based on prediction by genomic analysis, prior to functional analyses, its gene expression and protein localization were investigated by quantitative RT-PCR and immunohistochemical observation. Anti-cpnSET rabbit polyclonal serum prepared in this work was used to detect cpnSET protein in HEp-2 cells infected with $C$. pneumoniae J138. Simultaneously, anti- $C$. pneumoniae-specific monoclonal antibody RR402 and Hoechst 33258 were used for counter-staining. While chlamydial inclusions visualized by RR402 were detectable at any stages, cpnSET was detected only at 60 and 72 h postinfection (h.p.i.) (Fig. 2a) and in chlamydial cells (Fig. 2b). It is a similar expression pattern of $\mathrm{Hcl}$ and $\mathrm{Hc} 2$ proteins encoded by $h c t A$ and $h c t B$ genes, respectively (Hackstadt et al., 1991).

In chlamydial genomes, ftsK, yycJ and set genes are closely located in this order and seem to constitute an operon (Fig. 3a). In contrast to SET protein accumulation (Fig. 2a), chlamydial ftsK has been shown to express constantly from middle to late infection stages (Byrne et al., 2001) and our data from microarray analyses for chlamydial expression showed results consistent with this (data not shown). To clarify the expression of set, quantitative RT-PCR was performed. Relative expression of set, ompA, hctA and $h c t B$ normalized to $16 \mathrm{~S}$ rRNA amount are shown in Fig. 3(b). Constant expression of ompA and late-stage expression of $h c t A$ and $h c t B$ were consistent with previous reports (Fahr et al., 1995; Slepenkin et al., 2003). The accumulation of set mRNA was increased simultaneously with hctA and hctB after 54 h.p.i., suggesting that set may be transcriptionally independent of ftsK and regulated in the same manner as $h c t A$ and/or $h c t B$.

\section{CpnSET interaction with histone-like protein}

Physical interaction of cpnSET with $\mathrm{Hc} 1$ and Hc2 was tested by the yeast two-hybrid system. It was clarified that cpnSET can interact with $\mathrm{Hcl}$ and Hc2. Based on the experiments using deletion series of cpnSET, the binding to the Hc1 and Hc2 is through aa 137-200 and 137-160, respectively, of cpnSET, both of which regions contain substrate-binding amino acids required for histone methyltransferase activity (Fig. 4a). SET protein localization, expression stage and interaction with $\mathrm{Hcl}$ and $\mathrm{Hc} 2$ strongly suggest that cpnSET catalyses protein methylation to $\mathrm{Hcl}$ and $\mathrm{Hc} 2$ proteins. The original data for the yeast two-hybrid system are shown as Supplementary Fig. S2 with the online version of this paper.

To know whether cpnSET functions as a methyltransferase, an in vitro methyltransferase assay was carried out using recombinant murine histone $\mathrm{H} 3$ as a substrate under the conditions optimized for mouse G9a, which is a major mammalian histone methyltransferase containing a SET domain responsible for methylation of $\mathrm{K} 9$ in histone $\mathrm{H} 3$ at euchromatin (Tachibana et al., 2001). A $0.5 \mu \mathrm{g}$ sample of cpnSET, GST and G9a proteins was separately incubated with $0.5 \mu \mathrm{g}$ recombinant mouse histone $\mathrm{H} 3$. It was shown that cpnSET can methylate histone $\mathrm{H} 3$ and the methylation activity of cpnSET is $14 \%$ of G9a under these conditions (Fig. 4b).

As a substrate candidate of chlamydial proteins for cpnSET activity, $\mathrm{Hcl}$ protein was subjected to this assay with $\mathrm{Hc1}-1$, Hc1-2 and Hc1-3 (aa 1-50, 41-78 and 65-123, respectively). The full lengths of $\mathrm{Hc1}$ and Hc2 are difficult to keep soluble in the processes of protein purification and methyltransferase reaction. As a result, only the Hcl-1 was capable of being methylated by cpnSET (Fig. 4c). No apparently conserved sequences were found between $\mathrm{H} 3$ (aa 1-50) and Hc1-1 (Fig. 4c). However, the combination of two informatics analyses, structure modelling of cpnSET using the virus SET 
(a)

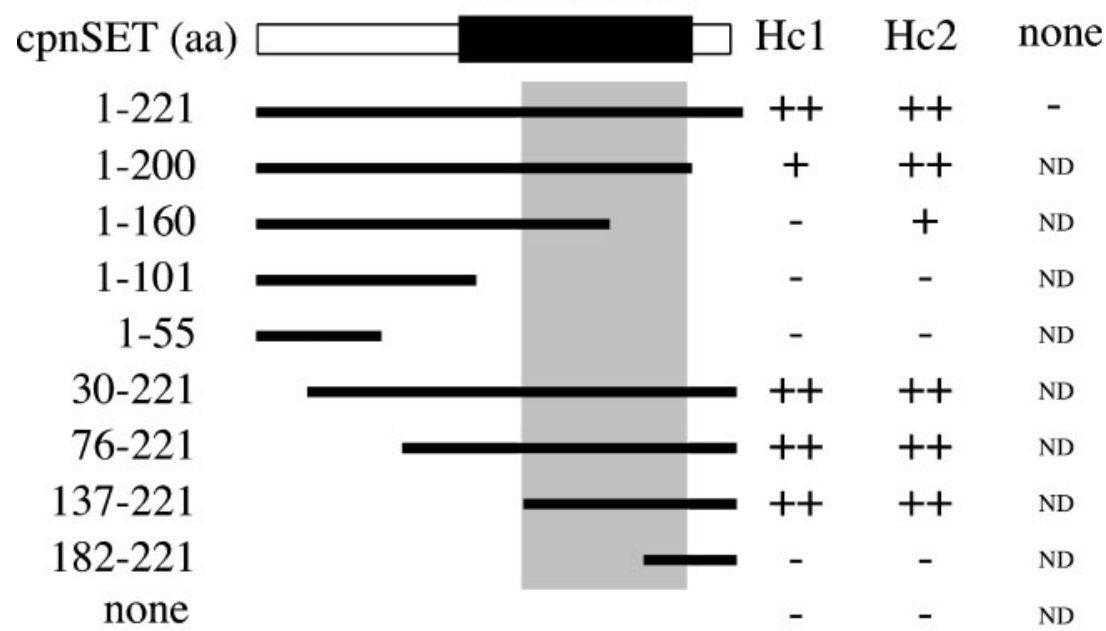

(b)

\section{G9a GST cpnSET}

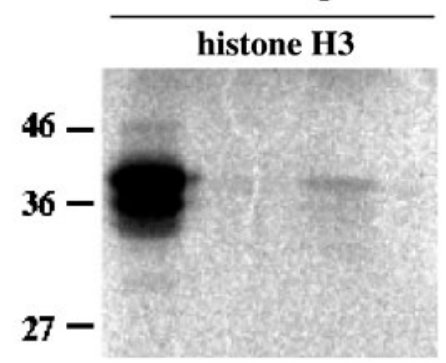

(c)

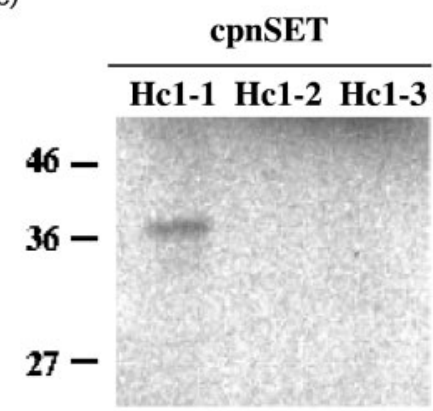

(d)

H3 $(1 \sim 50 \mathrm{aa}) \quad \begin{array}{llccr}1 & 10 & 20 & \text { ARTKQTARKSTGGKAPRKQLATKAARKSAPSTGGVKKPHRYRPGTVALRE }\end{array}$ Hc1-1 (1 50aa) MALKDTAKKMKDLLDSIQHDLAKAEKGNKAAAQRVRTDSIKLEKVAKLYR
Fig. 4. Interaction of cPnSET and chlamydial histone-like proteins. (a) Two-hybrid assay between cPnSET or its deletion series, and Hc1 and Hc2. The filled box (aa 102-181) and grey region show the SET domain and interaction domain of cPnSET with $\mathrm{Hc} 1$ and $\mathrm{Hc} 2$, respectively.,+++ and - , good, weak, and no growth, respectively, on culture medium without histidine. None, vector only; ND, not determined. (b) Histone H3 methylation. GST-G9a (indicated as G9a), GST-cpnSET (cpnSET) and GST protein (GST) were subjected to methylation of GST-H3 protein (histone H3). (c) Chlamydial $\mathrm{Hc} 1$ methylation. $\mathrm{Hc} 1$ deletion proteins $\mathrm{Hc} 1-$ 1, Hc1-2 and Hc1-3 were used as substrates for in vitro methylation by chlamydial GST-cpnSET (cpnSET). (d) Alignments of histone $\mathrm{H} 3$ and chlamydial histone-like protein, Hc1-1. Histone H3 (CAH73371) and Hc1-1 (NP_300943) are shown 50 aa from the $\mathrm{N}$ terminus. The predicted methylated residues are indicated with asterisks. structure and peptide docking analysis, indicates that $\mathrm{K} 27$ of $\mathrm{H} 3$, one of the specific methylation sites (Tachibana et al., 2001), and $\mathrm{K} 29$ of $\mathrm{Hc} 1$ were the best-fitting lysine residues to the catalytic space of cpnSET on the basis of the lowest docking energy. The modelled cpnSET structure and docking profile are shown in Supplementary Fig. S3 with the online version of this paper.

\section{DISCUSSION}

Numbers of genes encoding SET domain proteins have been identified in genomes of non-eukaryotic organisms, e.g. Paramecium bursaria chlorella virus-1, archaeal Methanosarcina mezei and bacterial Bacillus anthracis, Bacillus cereus, Xylella fastidiosa, Leptospira interrogans, Bradyrhizobium japonicum, Chlorobium tepidum and species of the family Chlamydiaceae. It has been reported that the viral SET domain protein methylates histone H3 Lys27, and the archaeal SET domain protein methylates lysine residues of eukaryotic histone $\mathrm{H} 4$ and archaeal DNA-binding protein MC1- $\alpha$ (Manzur et al., 2003; Manzur \& Zhou, 2005). Herein, cpnSET was shown to function as a protein methyltransferase to methylate both murine histone H3 and chlamydial $\mathrm{Hcl}$ in vitro, suggesting that cPnSET may play an important role in modification of $\mathrm{Hcl}$ proteins for the morphological change from RBs to EBs. Since localization of cpnSET was shown mainly in chlamydial cells, cpnSET may methylate $\mathrm{Hcl}$ in vivo, but it is still possible that chlamydial SET proteins are exported into host cells or that host histones are transported into chlamydial cells, and then cpnSET and host histones functionally interact with each other. Identification of physiological substrates for methylation by cpnSET and, if $\mathrm{Hcl}$ is one of the physiological substrates for methylation in vivo, elucidation of the significance of $\mathrm{Hcl}$ protein modification remains for further investigation. Here one of the eubacterial SET domain proteins was revealed as a protein methyltransferase, and this finding suggests that other eubacterial SET domain proteins may function as methyltransferases as well.

\section{ACKNOWLEDGEMENTS}

We thank Dr Yoichi Shinkai and Dr Makoto Tachibana for giving us plasmids (G9a, H3) and Mr Michael S. Patrick for help. This study was supported by Basic and Applied Research on Microbial Genome and 
Physiology, a Grant-in-Aid for Scientific Research (KAKEN-HI: 14770063, 16790225) and the National Project on Protein Structural and Functional Analyses from the Ministry of Education, Culture, Sports, Science and Technology of Japan.

\section{REFERENCES}

Alvarez-Venegas, R. \& Avramova, Z. (2002). SET-domain proteins of the $\mathrm{Su}(\mathrm{var}) 3-9, \mathrm{E}(\mathrm{z})$ and Trithorax families. Gene 285, 25-37.

Azuma, Y., Yamagishi, M. \& Ishihama, A. (1993). Subunits of the Schizosaccharomyces pombe RNA polymerase II: enzyme purification and structure of the subunit 3 gene. Nucleic Acids Res 21, 3749-3754.

Azuma, Y., Tabb, M. M., Vu, L. \& Nomura, M. (1995). Isolation of a yeast protein kinase that is activated by the protein encoded by SRP1 (Srplp) and phosphorylates Srplp complexed with nuclear localization signal peptides. Proc Natl Acad Sci U S A 92, 5159-5163.

Azuma, Y., Hirakawa, H., Yamashita, A., Cai, Y., Rahman, M. A., Suzuki, H., Mitaku, S., Toh, H., Goto, S. \& other authors (2006). Genome sequence of the cat pathogen, Chlamydophila felis. DNA Res 13, 15-23.

Barry, C. E., Hayes, S. F. \& Hackstadt, T. (1992). Nucleoid condensation in Escherichia coli that express a chlamydial histone homolog. Science 256, 377-379.

Beatty, W. L., Byrne, G. I. \& Morrison, R. P. (1993). Morphologic and antigenic characterization of interferon gamma-mediated persistent Chlamydia trachomatis infection in vitro. Proc Natl Acad Sci U S A 90, 3998-4002.

Belland, R. J., Nelson, D. E., Virok, D., Crane, D. D., Hogan, D., Sturdevant, D., Beatty, W. L. \& Caldwell, H. D. (2003). Transcriptome analysis of chlamydial growth during IFN-gammamediated persistence and reactivation. Proc Natl Acad Sci U S A 100, 15971-15976.

Byrne, G. I., Ouellette, S. P., Wang, Z., Rao, J. P., Lu, L., Beatty, W. L. \& Hudson, A. P. (2001). Chlamydia pneumoniae expresses genes required for DNA replication but not cytokinesis during persistent infection of HEp-2 cells. Infect Immun 69, 5423-5429.

Carlson, J. H., Porcella, S. F., McClarty, G. \& Caldwell, H. D. (2005). Comparative genomic analysis of Chlamydia trachomatis oculotropic and genitotropic strains. Infect Immun 73, 6407-6418.

Dillon, S. C., Zhang, X., Trievel, R. C. \& Cheng, X. (2005). The SETdomain protein superfamily: protein lysine methyltransferases. Genome Biol 6, 227.

Eswar, N., John, B., Mirkovic, N., Fiser, A., Ilyin, V. A., Pieper, U., Stuart, A. C., Marti-Renom, M. A., Madhusudhan, M. S. \& other authors (2003). Tools for comparative protein structure modeling and analysis. Nucleic Acids Res 31, 3375-3380.

Fahr, M. J., Douglas, A. L., Xia, W. \& Hatch, T. P. (1995). Characterization of late gene promoters of Chlamydia trachomatis. $J$ Bacteriol 177, 4252-4260.

Grieshaber, N. A., Grieshaber, S. S., Fischer, E. R. \& Hackstadt, T. (2006). A small RNA inhibits translation of the histone-like protein $\mathrm{Hcl}$ in Chlamydia trachomatis. Mol Microbiol 59, 541-550.

Hackstadt, T., Baehr, W. \& Ying, Y. (1991). Chlamydia trachomatis developmentally regulated protein is homologous to eukaryotic histone H1. Proc Natl Acad Sci U S A 88, 3937-3941.

Hahn, D. L., Dodge, R. W. \& Golubjatnikov, R. (1991). Association of Chlamydia pneumoniae (strain TWAR) infection with wheezing, asthmatic bronchitis, and adult-onset asthma. JAMA 266, 225-230.

Itzhaki, R. F., Wozniak, M. A., Appelt, D. M. \& Balin, B. J. (2004), Infiltration of the brain by pathogens causes Alzheimer's disease. Neurobiol Aging 25, 619-627.
Jones, S. R. \& Gelbart, W. M. (1993). The drosophila polycombgroup gene enhancer of zeste contains a region with sequence similarity to trithorax. Mol Cell Biol 13, 6357-6366.

Kalman, S., Mitchell, W., Marathe, R., Lammel, C., Fan, J., Hyman, R. W., Olinger, L., Grimwood, J., Davis, R. W. \& Stephens, R. S. (1999). Comparative genomes of Chlamydia pneumoniae and C. trachomatis. Nat Genet 21, 385-389.

Kouzarides, T. (2002). Histone methylation in transcriptional control. Curr Opin Genet Dev 12, 198-209.

Kuzmichev, A., Margueron, R., Vaquero, A., Preissner, T. S., Scher, M., Kirmizis, A., Ouyang, X., Brockdorff, N., Abate-Shen, C. \& other authors (2005). Composition and histone substrate of polycomb repressive group complexes change during cellular differentiation. Proc Natl Acad Sci U S A 102, 1859-1864.

Liu, H. Y., Badarinarayana, V., Audino, D. C., Rappsilber, J., Mann, M. \& Denis, C. L. (1998). The NOT proteins are part of the CCR4 transcriptional complex and affect gene expression both positively and negatively. EMBO J 17, 1096-1106.

Malinverni, R., Kuo, C. C., Campbell, L. A. \& Grayston, J. T. (1995). Reactivation of Chlamydia pneumoniae lung infection in mice by cortisone. J Infect Dis 172, 593-594.

Manzur, K. L. \& Zhou, M. M. (2005). An archaeal SET domain protein exhibits distinct lysine methyltransferase activity towards DNAassociated protein MC1-alpha. FEBS Let 579, 3859-3865.

Manzur, K. L., Farooq, A., Zeng, L., Plotnikova, O., Koch, A. W., Sachchidanand \& Zhou, M. M. (2003). A dimeric viral SET domain methyltransferase specific to Lys27 of histone H3. Nat Struct Biol 10, 187-196.

Marmorstein, R. (2003). Structure of SET domain proteins: a new twist on histone methylation. Trends Biochem Sci 28, 59-62.

Mehta, S. J., Miller, R. D., Ramirez, J. A. \& Summersgill, J. T. (1998). Inhibition of Chlamydia pneumoniae replication in HEp-2 cells by interferon-gamma: role of tryptophan catabolism. J Infect Dis 177, 1326-1331.

Miura, K., Inouye, S., Sakai, K., Takaoka, H., Kishi, F., Tabuchi, M., Tanaka, T., Matsumoto, H., Shirai, M. \& other authors (2001). Cloning and characterization of adenylate kinase from Chlamydia pneumoniae. J Biol Chem 276, 13490-13498.

Morris, G. M., Goodsell, D. S., Huey, R. \& Olson, A. J. (1996). Distributed automated docking of flexible ligands to proteins: parallel applications of AutoDock 2.4. J Comput Aided Mol Des 10, 293-304.

Perara, E., Ganem, D. \& Engel, J. N. (1992). A developmentally regulated chlamydial gene with apparent homology to eukaryotic histone H1. Proc Natl Acad Sci U S A 89, 2125-2129.

Rahman, M. A., Azuma, Y., Fukunaga, H., Murakami, T., Sugi, K., Fukushi, H., Miura, K., Suzuki, H. \& Shirai, M. (2005). Serotonin and melatonin, neurohormones for homeostasis, as novel inhibitors of infections by the intracellular parasite Chlamydia. J Antimicrob Chemother 56, 861-868.

Read, T. D., Brunham, R. C., Shen, C., Gill, S. R., Heidelberg, J. F. White, O., Hickey, E. K., Peterson, J., Utterback, T. \& other authors (2000). Genome sequences of Chlamydia trachomatis MoPn and Chlamydia pneumoniae AR39. Nucleic Acids Res 28, 1397-1406.

Read, T. D., Myers, G. S. A., Brunham, R. C., Nelson, W. C., Paulsen, I. T., Heidelberg, J., Holtzapple, E., Khouri, H., Federova, N. B. \& other authors (2003). Genome sequence of Chlamydophila caviae (Chlamydia psittaci GPIC): examining the role of niche-specific genes in the evolution of the Chlamydiaceae. Nucleic Acids Res 31, 2134-2147.

Rosenfeld, M. E., Blessing, E., Lin, T. M., Moazed, T. C., Campbell, L. A. \& Kuo, C. (2000). Chlamydia, inflammation, and atherogenesis. J Infect Dis 181 Suppl 3, S492-S497. 
Shirai, M., Hirakawa, H., Kimoto, M., Tabuchi, M., Kishi, F., Ouchi, K., Shiba, T., Ishii, K., Hattori, M. \& other authors (2000). Comparison of whole genome sequences of Chlamydia pneumoniae J138 from Japan and CWL029 from USA. Nucleic Acids Res 28, 2311-2314.

Slepenkin, A., Motin, V., de la Maza, L. M. \& Peterson, E. M. (2003). Temporal expression of Type III secretion genes of Chlamydia pneumoniae. Infect Immun 71, 2555-2562.

Stephens, R. S., Kalma, S., Lammel, C., Fan, J., Marathe, R., Aravind, L., Mitchell, W., Olinger, L., Tatusov, R. L. \& other authors (1998). Genome sequence of an obligate intracellular pathogen of humans: Chlamydia trachomatis. Science 282, 754-759.

Tachibana, M., Sugimoto, K., Fukushima, T. \& Shinkai, Y. (2001). SET-domain containing protein, G9a, is a novel lysine-preferring mammalian histone methyltransferase with hyperactivity and specific selectivity to lysines 9 and 27 of histone H3. J Biol Chem 276, 25309-25317.
Tao, S., Kaul, R. \& Wenman, W. M. (1991). Identification and nucleotide sequence of a developmentally regulated gene encoding a eukaryotic histone H1-like protein from Chlamydia trachomatis. J Bacteriol 173, 2818-2822.

Thomson, N. R., Yeats, C., Bell, K., Holden, M. T., Bentley, S. D., Livingstone, M., Cerdeno-Tarrago, A. M., Harris, B., Doggett, J. \& other authors (2005). The Chlamydophila abortus genome sequence reveals an array of variable proteins that contribute to interspecies variation. Genome Res 15, 629-640.

Xiao, B., Wilson, J. R. \& Gamblin, S. J. (2003). SET domains and histone methylation. Curr Opin Struct Biol 13, 699-705.

Zhang, X., Yang, Z., Khan, S. I., Horton, J. R., Tamaru, H., Selker, E. U. \& Cheng, X. (2003). Structural basis for the product specificity of histone lysine methyltransferases. Mol Cell 12, 177-185.

Edited by: J. Parkhill 